Check for updates

Cite this: Phys. Chem. Chem. Phys., 2019, 21, 10402

Received 25th February 2019 Accepted 1st May 2019

DOI: $10.1039 / c 9 c p 01114 \mathrm{e}$

rsc.li/pccp

\title{
Hydrogen quenches the size effects in carbon clusters $\uparrow$
}

\begin{abstract}
José I. Martínez (D) *a and Julio A. Alonso (D) ${ }^{b}$
A characteristic fingerprint of atomic clusters is that their properties can vary in a non-smooth way with the cluster size $N$. This is illustrated herein by studying the cluster size dependence of several properties of neutral $\mathrm{C}_{N}$ and cationic $\mathrm{C}_{N}^{+}$carbon clusters: $\mathrm{C}-\mathrm{C}$ bond lengths, cluster structure, intrinsic cluster stability, ionization energy, and spatial distribution of the reactivity index for charge exchange with electrophiles. Nonetheless, clusters can lose the size dependence of their properties by interaction with other chemical species, which is rationalized in this study by analyzing carbon clusters fully saturated with hydrogen to form linear alkanes, $\mathrm{C}_{N} \mathrm{H}_{2 N+2}$. In all cases, the lowest energy structures are zigzagging linear chains, the variations of $\mathrm{C}-\mathrm{C}$ bond lengths and $\widehat{\mathrm{CCC}}$ angles with alkane size are very minor and smooth, the stability function shows practically no structure as a function of the alkane size, the ionization energies just decrease smoothly with alkane size, and the spatial distribution of the reactivity index is analogous and highly delocalized in all the alkanes. In summary, the interaction of carbon clusters with hydrogen to form alkanes quenches all the size-dependent features that the carbon clusters originally owned. The arrival at the quenching of the size effects follows an involved path. In each $\mathrm{C}_{N} \mathrm{H}_{n}$ family with fixed $N$, the values of the properties of the molecules like the ionization potential, the electron affinity, and others show sizable oscillations as the number of hydrogen atoms grows from the pure carbon cluster to the alkane.
\end{abstract}

\section{Introduction}

The most important characteristic of atomic clusters and nanoparticles is the non-smooth dependence of their properties with the cluster size, that is, with the number of atoms in the cluster. This non-smooth variation has been detected in the physical and chemical properties. As an illustration, a few noteworthy cases can be selected among the many possible examples.

The structures of the clusters often vary with the cluster size. As a remarkable example, the structures of cationic boron clusters $\mathrm{B}_{N}^{+}$with sizes between 17 and 25 atoms are short cylinders formed by two rings, whereas smaller clusters are quasiplanar. ${ }^{1}$ On the other hand, the main allotrope of bulk solid boron, rhombohedral $\alpha-\mathrm{B}$, is formed by icosahedral $\mathrm{B}_{12}$ clusters connected by strong covalent bonds. ${ }^{2}$ Electronic properties are also quite sensitive to cluster size. The ionization energy of alkali metal clusters presents sudden drops at the

\footnotetext{
${ }^{a}$ Materials Science Factory, Dept. Surfaces, Coatings and Molecular Astrophysics, Institute of Material Science of Madrid (ICMM-CSIC), Sor Juana Inés de la Cruz 3, ES-28049 Madrid, Spain.E-mail: joseignacio.martinez@icmm.csic.es

${ }^{b}$ Departamento de Física Teórica, Atómica y Óptica, University of Valladolid, ES-47011 Valladolid, Spain

$\uparrow$ The authors dedicate this work to Professor Pablo Espinet on the occasion of his 70th birthday.
}

magic numbers $N=8,20,40,58,92, \ldots,{ }^{3}$ which are explained by the high stability achieved by the closing of electronic shells that occurs at these particular cluster sizes. ${ }^{4,5}$ The binding energies of atomic core orbitals can be very sensitive to the size of the nanoparticles and can be probed by X-ray absorption near-edge structure (XANES) spectroscopy. ${ }^{6}$ Mercury clusters reveal a band gap closure with increasing cluster size, which corresponds to an insulator-metal transition. ${ }^{7}$ Optical properties represent another interesting case. The color of gold nanorods depends on their aspect ratio (ratio between the lengths of the longitudinal and transverse axes of the nanorod), because the aspect ratio influences the optical absorption spectrum. ${ }^{8}$ Magnetism at the nanoscale is intricate and rich in surprises. For instance, the magnetic moment per atom of nickel clusters shows an overall decrease as a function of cluster size, but oscillations are superimposed to that decrease, with deep local minima at $\mathrm{Ni}_{6}$ and $\mathrm{Ni}_{13}$, and another minimum at $\mathrm{Ni}_{55}-\mathrm{Ni}_{56}{ }^{9}$ Larger nanoparticles also display a substantial dependence of the magnetization with temperature and particle volume. For instance, a temperature-induced ferromagnetic to superparamagnetic transition has been observed in $\mathrm{Fe}$ nanoparticles only below a threshold size. ${ }^{10}$ Following the discovery of the catalytic properties of small gold nanoparticles by Haruta and coworkers, ${ }^{11}$ one of the areas where the cluster size effects show more promise is nanocatalysis. ${ }^{12}$ In their seminal work 
Haruta and coworkers investigated the role of gold nanoparticles on the direct oxidation of propene by a mixture of $\mathrm{O}_{2}$ and $\mathrm{H}_{2}$, and observed that the selectivity towards the final product is highly sensitive to the size and shape of the gold particles. The adsorption energies of $\mathrm{O}, \mathrm{O}_{2}, \mathrm{OH}, \mathrm{OOH}, \mathrm{H}_{2} \mathrm{O}, \mathrm{H}_{2} \mathrm{O}_{2}$ and $\mathrm{CO}$ on the surface of $\mathrm{Pt}$ and $\mathrm{Au}$ clusters and nanoparticles depend sensitively on the cluster size, and the convergence to the bulk occurs faster for Pt. ${ }^{13,14}$

Magic numbers (magic sizes) are a characteristic of clusters, and the numerical values of the cluster properties usually show noticeable changes after those magic numbers, as mentioned above for the ionization potentials of metal clusters. The magic numbers often arise from the closing of shells, either electronic shells ${ }^{4,5}$ or atomic shells, ${ }^{15}$ or to specially favorable electronic and atomic structures like in $\mathrm{C}_{60} \cdot{ }^{16}$ Magic numbers have been also observed in the case of clusters synthesized on metal surfaces. Artaud et al. ${ }^{17}$ have recently reported the discovery of a novel family of metastable non-Kekule carbon clusters of magic sizes and morphologies that covalently anchor to the substrate preventing the growth of graphene.

The selected examples reviewed above give evidence of the variety of size effects occurring in clusters. An interesting topic seldom studied is the quenching of the size effects in clusters. This can occur by the interaction of the clusters with other species, and in this work we focus on the quenching of the size effects in small carbon clusters by hydrogen. For this purpose we have considered a family of molecules with a similar structure, the linear alkanes $\mathrm{C}_{N} \mathrm{H}_{2 N+2}$ with $N=2-25$. In Section 2 we briefly present the computational method used in the calculations. The results showing how the presence of $\mathrm{H}$ atoms quenches the size effects are reported in Sections 3.1 and 3.2. The alkane $\mathrm{C}_{N} \mathrm{H}_{2 N+2}$ is the heaviest molecule at the end of each $\mathrm{C}_{N} \mathrm{H}_{n}$ family, and the variation of the properties as the number $n$ of hydrogen atoms increases in the family is analyzed in Section 3.3. Finally, the conclusions are summarized in Section 4.

\section{Computational approach}

The atomic structures, cluster stabilities, electronic properties and reactivity of neutral $\mathrm{C}_{N}$ and cationic $\mathrm{C}_{N}^{+}$carbon clusters with $N \leq 16$, and neutral $\mathrm{C}_{N} \mathrm{H}_{2 N+2}$ and cationic $\left(\mathrm{C}_{N} \mathrm{H}_{2 N+2}\right)^{+}$ alkanes with $N=2-25$ have been investigated using the density functional theory as implemented in the Gaussian09 atomistic simulation package. ${ }^{18}$ The hybrid B3LYP method $^{19,20}$ was employed to treat exchange and correlation effects, and the molecular orbitals were expanded on a cc-PVQZ basis ${ }^{21}$ set. In the case of the pure carbon clusters, for each cluster-size we have used a number of different isomers reported in the literature as starting geometries for the structural optimization within the B3LYP method. ${ }^{22}$ Besides, for all the neutral $\mathrm{C}_{N}$ and cationic $\mathrm{C}_{N}^{+}$isomers studied we have analyzed the two lowestlying electronic spin states. On the other hand, alkanes are hydrocarbon compounds consisting of $\mathrm{C}$ and $\mathrm{H}$ atoms. Their chemical structure consists of single bonds only. Three types of alkanes exist: linear, branched and cyclic. In the present study we focus on the linear alkanes $\mathrm{C}_{N} \mathrm{H}_{2 N+2}$ : in these molecules all the carbon atoms have $\mathrm{sp}^{3}$ hybridization, form a zigzagging chain-like structure and are fully saturated with $\mathrm{H}$ atoms. Starting with the most simple case of ethane, $\mathrm{C}_{2} \mathrm{H}_{6}$, the initial geometries for the geometrical optimizations of neutral $\mathrm{C}_{N} \mathrm{H}_{2 N+2}$ and cationic $\left(\mathrm{C}_{N} \mathrm{H}_{2 N+2}\right)^{+}$alkanes have been constructed by sequentially adding internal $-\mathrm{CH}_{2}-$ units between the two end $-\mathrm{CH}_{3}$ groups.

Reactivity indexes introduced by Fukui et l. $^{23}$ have been computed for the $\mathrm{C}_{N}$ and $\mathrm{C}_{N} \mathrm{H}_{2 N+2}$ clusters. The Fukui functions provide a measure of the local reactivity of a molecule. In the framework of density functional theory the Fukui function

$$
f(\mathbf{r})=\left[\frac{\delta \mu}{\delta \nu(\mathbf{r})}\right]_{N}=\left[\frac{\partial \rho(\mathbf{r})}{\partial N}\right]_{\nu}
$$

provides a quantitative measure of the change of the chemical potential $\mu$ of a system in response to a local change $\delta \nu(\mathbf{r})$ in the external potential $\nu(\mathbf{r})$ (due to the presence of a reagent species) for fixed number $N$ of electrons. The function $f(\mathbf{r})$ can also be viewed as the local change of the electron density due to a global change in the number of electrons for fixed external potential. It is useful to consider the Fukui functions from above, $f^{+}(\mathbf{r})$, and from below, $f^{-}(\mathbf{r}),{ }^{24}$ that is

$$
f(\mathbf{r})^{ \pm}=\left[\frac{\partial \rho(\mathbf{r})}{\partial N}\right]_{\nu}^{ \pm},
$$

which measure the change in the local electron density as the number of electrons changes from $N$ to $N+\mathrm{d} N$ or to $N-\mathrm{d} N$, respectively. In the present case we are interested in $f^{-}(\mathbf{r})$. In practice, $f^{-}(\mathbf{r})$ can be computed, by using a finite difference approximation, as the density difference: ${ }^{25,26}$

$$
f^{-}(\mathbf{r})=\rho_{\nu, N}(\mathbf{r})-\rho_{\nu, N-1}(\mathbf{r})
$$

where $\rho_{\nu, N}(\mathbf{r})$ and $\rho_{\nu, N-1}(\mathbf{r})$ are the ground-state electronic densities of the neutral and cationic molecules in the geometrical configuration of the neutral.

\section{Results and discussion}

\subsection{Size effects in small carbon clusters}

The lowest-energy structures of neutral $\mathrm{C}_{N}$ and cationic $\mathrm{C}_{N}^{+}$ carbon clusters with $N=2-16$ calculated by the B3LYP method have been discussed in a previous work ${ }^{22}$ and here we only summarize the results. Neutral $\mathrm{C}_{2}-\mathrm{C}_{10}$ form cumulenic linear structures with nearly equal bond lengths, and $\mathrm{C}_{11}-\mathrm{C}_{16}$ exhibit monocyclic-ring structures. For $\mathrm{C}_{4}, \mathrm{C}_{6}$ and $\mathrm{C}_{8}$ we found lowlying cyclic isomers with energies differing by less than $0.15 \mathrm{eV}$ from the linear structures. Concerning the cationic clusters, $\mathrm{C}_{2}^{+}$, $\mathrm{C}_{4}^{+}-\mathrm{C}_{7}^{+}$and $\mathrm{C}_{9}^{+}$are linear, and $\mathrm{C}_{10}^{+}-\mathrm{C}_{16}^{+}$exhibit monocyclic-ring structures. The ground state structure of $\mathrm{C}_{3}^{+}$is a bent configuration, $0.19 \mathrm{eV}$ more stable than the perfect linear isomer. $\mathrm{C}_{8}^{+}$ has a cyclic ground-state configuration and a low-lying linear isomer nearly degenerate. Finally, $\mathrm{C}_{6}^{+}$is a slightly non-planar distorted hexagon with practically the same energy as the linear form. 


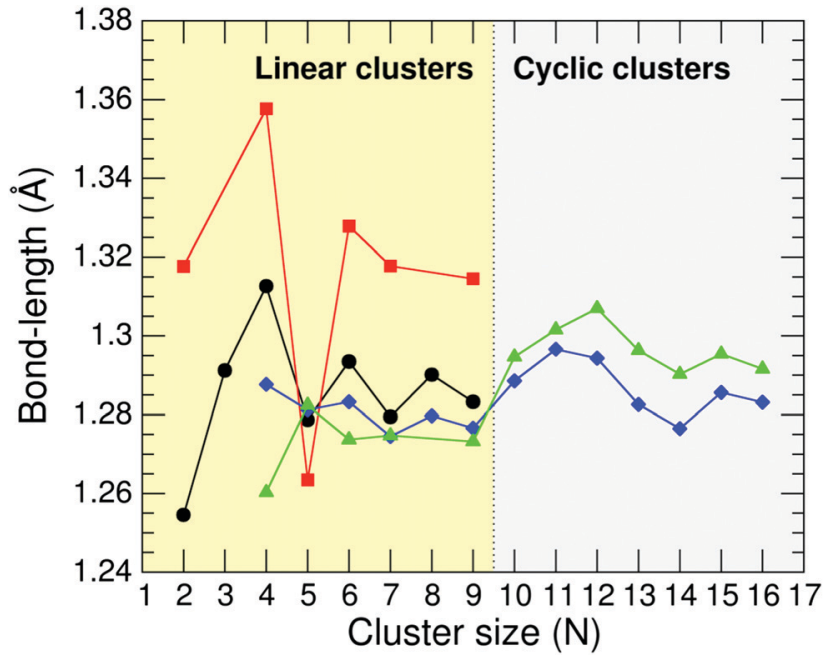

Fig. $1 \mathrm{C}-\mathrm{C}$ bond-lengths (in $\AA$ ) in neutral $\mathrm{C}_{N}$ and cationic $\mathrm{C}_{N}^{+}$carbon clusters from $N=2$ to 16 . $C-C$ bond-length at the edges of the linear $C_{N}$ clusters (black circles), and $\mathrm{C}_{N}^{+}$clusters (red squares). Average $\mathrm{C}-\mathrm{C}$ bondlengths for $C_{N}$ and $C_{N}^{+}$clusters (blue diamonds and green triangles, respectively). The average in the cyclic clusters is over all $\mathrm{C}-\mathrm{C}$ bonds. In the linear clusters, the average is over the internal bonds excluding the two $\mathrm{C}-\mathrm{C}$ bonds at the cluster ends.

Fig. 1 shows different curves of $\mathrm{C}-\mathrm{C}$ bond-length values for neutral $\mathrm{C}_{N}$ and cationic $\mathrm{C}_{N}^{+}$carbon clusters from $N=2$ to 16 . The diamonds (blue curve) and triangles (green curve) report the average $\mathrm{C}-\mathrm{C}$ bond lengths for $\mathrm{C}_{N}$ and $\mathrm{C}_{N}^{+}$, respectively. The average in the cyclic clusters is over all $\mathrm{C}-\mathrm{C}$ bonds, but in the linear clusters the average is over the internal bonds excluding the two $\mathrm{C}-\mathrm{C}$ bonds at the cluster ends. The circles (black curve) and squares (red curve) correspond to $\mathrm{C}-\mathrm{C}$ bond lengths at the ends of the linear $\mathrm{C}_{N}$ and $\mathrm{C}_{N}^{+}$clusters, respectively. The reason for distinguishing between internal $\mathrm{C}-\mathrm{C}$ bonds and $\mathrm{C}-\mathrm{C}$ bonds at the cluster ends in linear clusters is that the internal $\mathrm{C}$ atoms have two $\mathrm{C}$ neighbors, while the $\mathrm{C}$ atoms at the ends of the cluster only have one neighbor. It is worth noticing that all the bond length curves display an irregular and oscillating trend. This effect is more pronounced for the smallest clusters with a linear arrangement.

In addition to the transition from linear to cyclic structures and the oscillations of the bond lengths, these clusters also show a pronounced size dependence of other properties. The cluster stability, measured by the cluster binding energy, has an interesting oscillating behavior as a function of cluster size. ${ }^{22}$ But the best way to appreciate the variations of the cluster stability is through the stability function. Fig. 2 shows the stability functions $\Delta_{2}\left(\mathrm{C}_{N}\right)$ and $\Delta_{2}\left(\mathrm{C}_{N}^{+}\right)$of neutral and cationic carbon clusters, respectively. The stability function of the neutral clusters is defined

$$
\Delta_{2}\left(\mathrm{C}_{N}\right)=E\left(\mathrm{C}_{N+1}\right)+E\left(\mathrm{C}_{N-1}\right)-2 E\left(\mathrm{C}_{N}\right)
$$

where $E\left(\mathrm{C}_{N}\right)$ represents the total energy of the cluster with $N$ atoms, and a similar definition applies to the cationic clusters. The functions $\Delta_{2}\left(\mathrm{C}_{N}\right)$ and $\Delta_{2}\left(\mathrm{C}_{N}^{+}\right)$measure the stability of the cluster of size $N$ compared to the averaged energy of the clusters

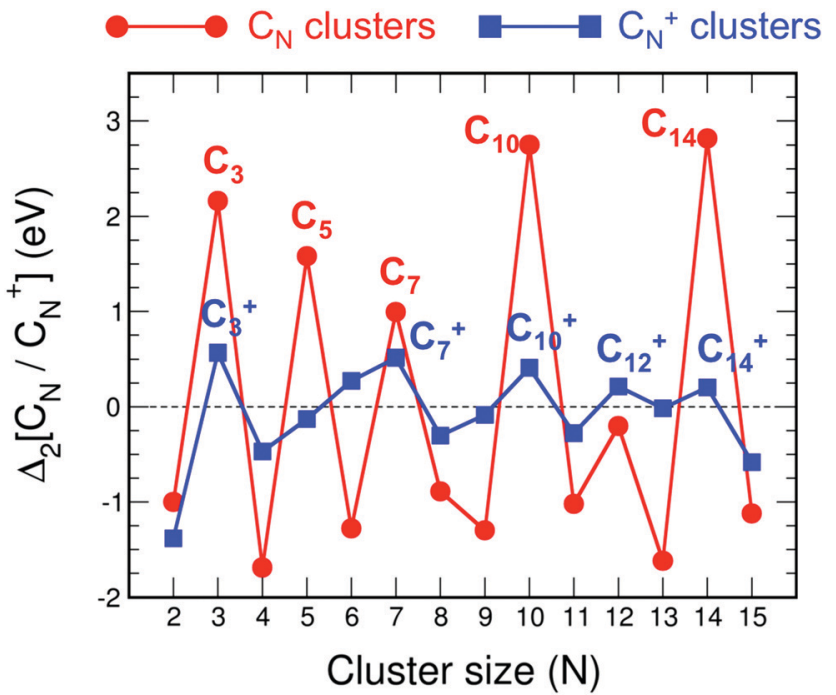

Fig. 2 Stability functions $\Delta_{2}\left(C_{N}\right)$ and $\Delta_{2}\left(C_{N}^{+}\right)$of neutral (red) and cationic (blue) carbon clusters up to $N=15$.

with neighbor sizes, $N+1$ and $N-1$. Clusters more stable than the neighbors are characterized by positive peaks in a plot of the stability function versus $N$. Fig. 2 indeed reveals that $\Delta_{2}\left(\mathrm{C}_{N}\right)$ and $\Delta_{2}\left(\mathrm{C}_{N}^{+}\right)$possess a rich structure and noticeable cluster size effects, with pronounced stability peaks for particular cluster sizes. In a previous work ${ }^{22}$ we have shown that the details of the succession of high and low values of the stability function compare very well with the local variations of the cluster abundance measured by mass spectrometry in cluster beams produced by pulsed laser vaporization of graphite. ${ }^{27}$ It is interesting to notice the energy scale of Fig. 2, that is, the range of values of $\Delta_{2}\left(\mathrm{C}_{N}\right)$ and $\Delta_{2}\left(\mathrm{C}_{N}^{+}\right)$. Those values vary between $-2 \mathrm{eV}$ and $3 \mathrm{eV}$.

The ionization energy IP of carbon clusters also reveals clear size effects. Fig. 3 shows our calculated ionization energies of the carbon clusters $\mathrm{C}_{N}$ with $N$ up to 15 compared to the values measured by photoionization spectroscopy by Belau et al., ${ }^{27}$ and to results of highly accurate coupled cluster (CC) calculations, also performed by Belau et al. ${ }^{27}$ for clusters up to $N=10$. We only provide a short discussion of the main features because Belau et al. have presented a detailed and comprehensive analysis of the comparison between the experiments and the $\mathrm{CC}$ results. One can notice the smaller values of the experimental IPs compared to the CC and B3LYP calculations up to $\mathrm{C}_{6}$. This difference has been ascribed by Belau $e$ al. to the likely presence of clusters in electronic excited states in their experiments. In fact, charge exchange $\mathrm{e}^{28,29}$ and electron impact experiments $^{30}$ yielded values in better accord with the theoretical results of Fig. 3. The agreement between theory and experiment is quite satisfactory for $\mathrm{C}_{7}$ to $\mathrm{C}_{9}$. Finally, the comparison is less satisfactory for $N$ larger than 9 , where again the theoretical IPs are higher than the experimental ones (for $\mathrm{C}_{10}, \mathrm{C}_{12}, \mathrm{C}_{14}$ and $\mathrm{C}_{15}$ ) in a size-range where the occurrence on clusters in excited electronic states is less likely. In this size region the IPs from electron impact experiments are even lower, 


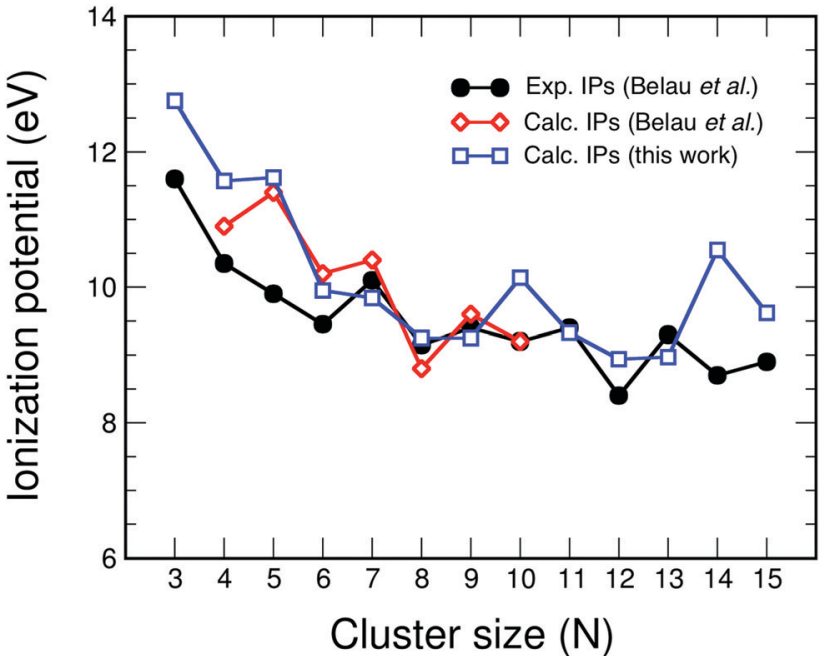

Fig. 3 Comparison between experimental (black circles) ${ }^{27}$ and calculated adiabatic ionization energies (in $\mathrm{eV}$ ) of carbon clusters. Two sets of calculations are included: results from coupled cluster CC calculations (red diamonds), ${ }^{27}$ and B3LYP results (this work; blue squares).

and the trend of alternation of local maxima and minima is shifted with respect to the alternation seen in the photoionization experiments; that is, low IP values in the photoionization experiment correspond to high IP values in the electron impact experiments, and vice versa. In spite of the differences between experiment and theory in Fig. 3, which should deserve a further study, the message we want to extract from this figure, that is relevant for the present work, is that IP varies with the cluster size in a non-smooth way, that is, IP shows pronounced cluster size effects.

\subsection{Quenching of the size effects by hydrogen}

The remarkable size effects observed in the stability, ionization energies and C-C bond lengths of $\mathrm{C}_{N}$ and $\mathrm{C}_{N}^{+}$clusters can be quenched by functionalizing the clusters. To investigate this issue we consider the functionalization of the carbon clusters with hydrogen by forming linear alkanes, with composition $\mathrm{C}_{N} \mathrm{H}_{2 N+2}$, in which all the $\mathrm{C}-\mathrm{C}$ bonds are single bonds. The $\mathrm{C}_{3} \mathrm{H}_{8}$ and $\mathrm{C}_{16} \mathrm{H}_{34}$ alkanes are shown in Fig. 4. Neutral and cationic linear alkanes have been studied.

The $\mathrm{C}-\mathrm{C}$ bond lengths in the neutral alkanes range, for all sizes studied, between $1.529 \AA$ and $1.535 \AA$ and the $\widehat{\mathrm{CCC}}$ angles in the carbon chains range between $113.4^{\circ}$ and $113.8^{\circ}$.

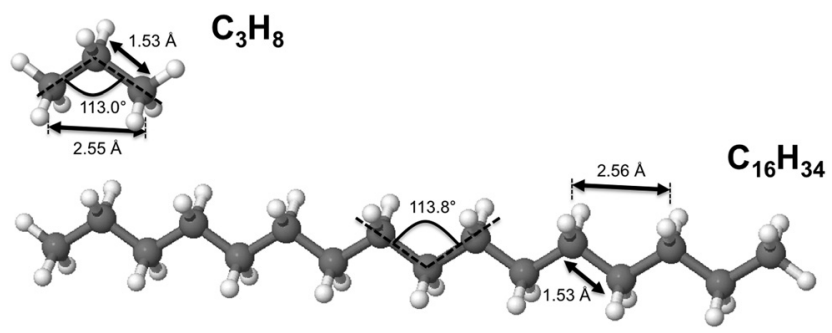

Fig. 4 Computed gas-phase structure of the $\mathrm{C}_{3} \mathrm{H}_{8}$ and $\mathrm{C}_{16} \mathrm{H}_{34}$ alkanes.
The variation in the $\mathrm{C}-\mathrm{C}$ bond lengths, $0.006 \AA$ is negligible in the scale of bond length changes observed in Fig. 1 for pure carbon clusters. This observation puts in evidence the quenching of structural effects by hydrogen functionalization. Interestingly, the quenching in the case of the cationic alkanes is less drastic. Fig. 5 reports $\mathrm{C}-\mathrm{C}$ bond-lengths and $\widehat{\mathrm{CCC}}$ angles for the ground state structures of cationic $\left(\mathrm{C}_{N} \mathrm{H}_{2 N+2}\right)^{+}$alkanes up to $N=25$. The squares (red curve) shown in the left panel of Fig. 5 as a function of $N$ represent the average $\mathrm{C}-\mathrm{C}$ bond lengths, where that average is taken over the all the bonds excluding the two $\mathrm{C}-\mathrm{C}$ bonds at the alkane ends. The circles (red curve) correspond to the $\mathrm{C}-\mathrm{C}$ bond lengths at the ends of the alkane. The angles, also separated in two curves, are given in the right panel of Fig. 5. The diamonds (blue curve) represent the $\widehat{\mathrm{CCC}}$ angles at the end of the alkane structure, and the triangles (green curve) are averages over all the $\widehat{\mathrm{CCC}}$ angles excluding the angles at the end of the alkane chain. It is noticeable that abrupt changes in the variation of the $\widehat{\mathrm{CCC}}$ angles with $N$ are quenched starting from $N=5$. Beyond $N=5$, a monotonic and smooth variation towards a limiting value of the angle is observed. In a similar way, the bond lengths vary monotonically starting from $N=5$ (red curve) and $N=7$ (black curve). This quenching of size effects contrasts with the nonsmooth and non-monotonic behavior displayed by the $\mathrm{C}-\mathrm{C}$ bond lengths of the pure carbon clusters in Fig. 1. In this respect, one should notice, however, that the cationic alkanes are more resilient than the neutrals.

The calculated stability functions of neutral $\mathrm{C}_{N} \mathrm{H}_{2 N+2}$ and cationic $\left(\mathrm{C}_{N} \mathrm{H}_{2 N+2}\right)^{+}$alkanes have been plotted as a function of $N$ in Fig. 6. In the case of neutral alkanes the stability function is defined as

$$
\Delta_{2}(N)=E(N+1)+E(N-1)-2 E(N)
$$

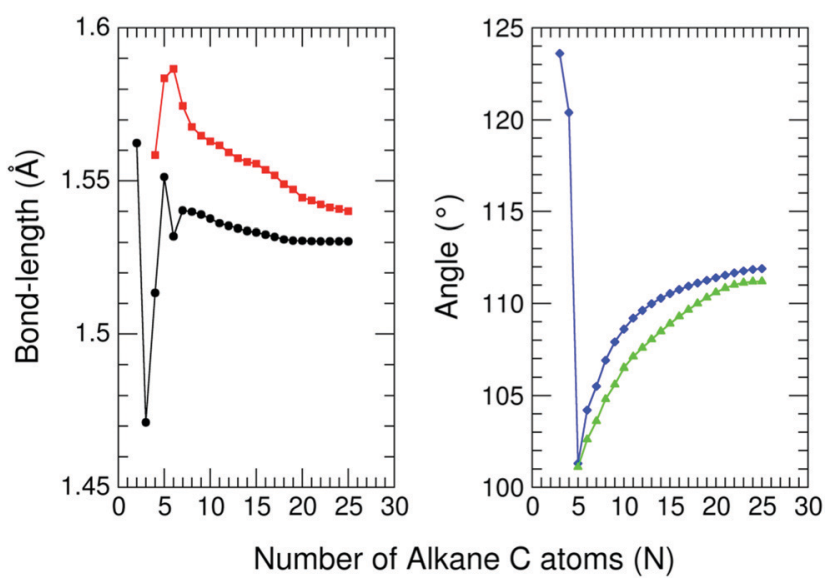

Fig. $5 \mathrm{C}-\mathrm{C}$ bond-lengths (in $\AA$ ) and $\widehat{\mathrm{CCC}}$ angles (in degrees) for cationic $\left(\mathrm{C}_{N} \mathrm{H}_{2 N+2}\right)^{+}$alkanes in their ground-state structures as a function of $N$ up to $N=25$. Left panel: Squares (red curve) represent the average $\mathrm{C}-\mathrm{C}$ bond lengths, where that average is taken over the all the bonds excluding the two $\mathrm{C}-\mathrm{C}$ bonds at the alkane ends; circles (red curve) correspond to the $\mathrm{C}-\mathrm{C}$ bond lengths at the ends of the alkane. Right panel: Diamonds (blue curve) represent the $\widehat{\mathrm{CCC}}$ angles at the end of the alkane structure, and the triangles (green curve) are averages over all the $\widehat{C C C}$ angles excluding those at the end of the alkane chain. 


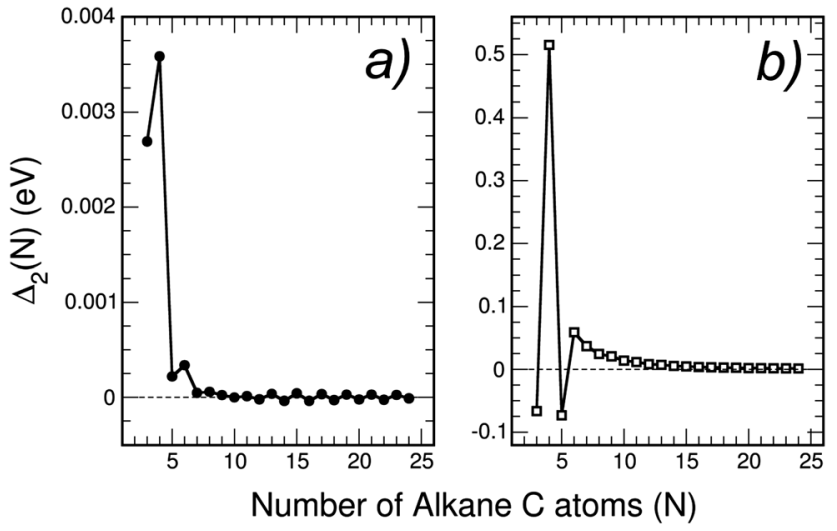

Fig. 6 Stability function $\Delta_{2}(N)$ (in eV) of: (a) neutral alkanes $\mathrm{C}_{N} \mathrm{H}_{2 N+2}$, and (b) cationic alkanes $\left(\mathrm{C}_{N} \mathrm{H}_{2 N+2}\right)^{+}$for $N=3-24$. Notice that the scale of energies has been enlarged by a factor of $\times 1000$ with respect to Fig. 1 in the case of neutral alkanes, and by a factor of $\times 10$ for cationic alkanes.

where $N$ indicates the number of carbon atoms in the alkane, and $E(N)$ represents the energy of the $\mathrm{C}_{N} \mathrm{H}_{2 N+2}$ alkane. Because the linear alkanes have one $\mathrm{CH}_{3}$ group at each of the two ends of the chain, the alkane with $N$ carbon atoms has $N-2$ inner $\mathrm{CH}_{2}$ groups.

Using in Fig. 6 an energy scale like that in Fig. 2, the plot of $\Delta_{2}(N)$ for neutral alkanes is just a horizontal line indistinguishable from the horizontal axis, that is $\Delta_{2}(N)=0$ independent of size $N$. This means that the size effects observed in Fig. 2 for pure neutral carbon clusters have been completely quenched by the functionalization with hydrogen in the linear alkanes. Fig. 6 shows that by enlarging the energy scale in a factor of $\times 1000$, that is, focusing on a meV scale, faint size effects can be appreciated in $\Delta_{2}(N)$, which oscillates with $N$. The oscillations are extremely weak, and only noticeable for $N$ lower than 7 . In the case of cationic alkanes the energy scale has to be enlarged by only a factor of $\times 10$ in order to appreciate faint size effects. Again, those faint size effects are restricted to $N$ lower than 7 , with stability maxima appearing at $N=4$ and $N=6$ (also in the neutral case), and the oscillations have totally disappeared for $N$ larger than 7 .

The calculated ionization potentials

$$
\mathrm{IP}(N)=E\left(\mathrm{C}_{N} \mathrm{H}_{2 N+2}\right)^{+}-E\left(\mathrm{C}_{N} \mathrm{H}_{2 N+2}\right)
$$

of linear alkanes are shown in Fig. 7. Experimental ionization potentials, ${ }^{31}$ available for $N=3-11$, are also included, and the comparison of calculated and experimental IPs is satisfactory. The calculated IPs are slightly smaller than the experimental IPs, with differences quite constant (except for $\mathrm{C}_{3} \mathrm{H}_{8}$ ), and in all cases below 5\%. Comparison with the calculations of other authors $^{32}$ is also satisfactory. The ionization potential of the linear alkanes decreases with $N$ but the decrease is smooth, and in contrast to the behavior of IP for the pure carbon clusters (see Fig. 3), abrupt size effects have been completely quenched in the linear alkanes by the effect of hydrogen. The values of IP have not yet reached a saturation value for the largest alkanes studied here, $\mathrm{C}_{25} \mathrm{H}_{52}$. The calculated electron affinities of the

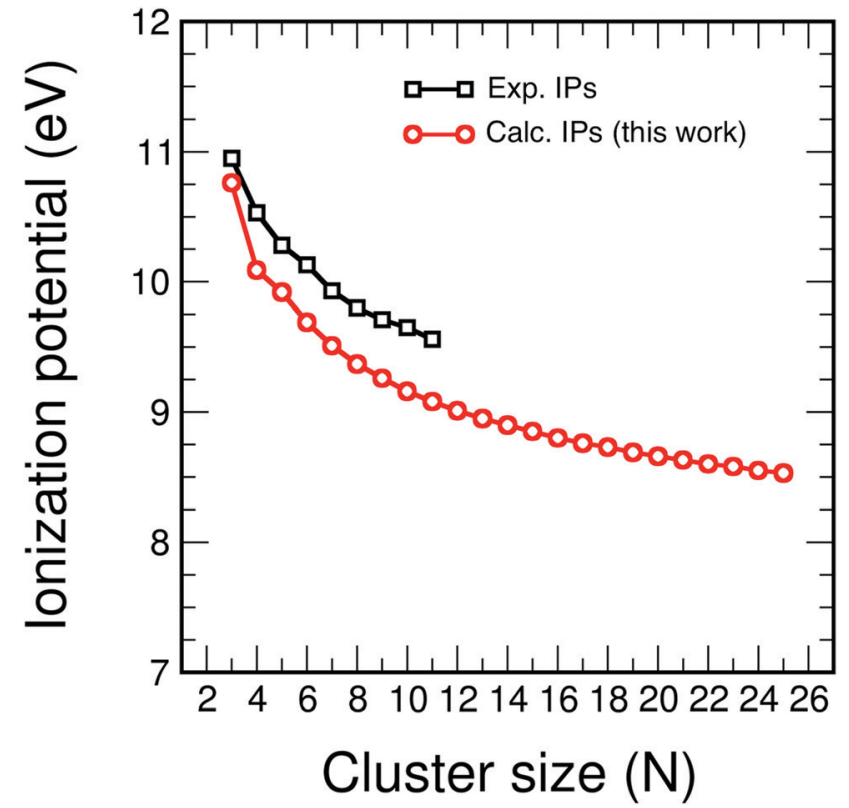

Fig. 7 Calculated ionization potentials (in eV) of $\mathrm{C}_{N} \mathrm{H}_{2 N+2}$ alkenes (red circles), compared to available experimental ionization potentials (black squares). ${ }^{31}$

alkanes are negative; that is, the linear alkane anions are not stable against electron detachment. This is in contrast to pure carbon anions. ${ }^{33}$

The large ionization potentials and negative electron affinities of the alkanes are consistent with the well-known low reactivity of these molecules with ionic and other polar substances. The chemical reactivity is often studied by using the Fukui functions. ${ }^{23}$ As noticed above in Section 2, in a density functional framework, a good approximation to the Fukui function measuring the local reactivity of a molecule towards (electron-poor) electrophiles is given by the electron density difference between the neutral and the cationic molecules. ${ }^{25,26}$ For the case of carbon clusters and alkanes, respectively,

$$
\begin{gathered}
f_{\mathrm{C}_{N}}^{-}(\mathbf{r})=\rho_{\mathrm{C}_{N}}(\mathbf{r})-\rho_{\mathrm{C}_{N}^{+}}(\mathbf{r})=\Delta \rho_{\mathrm{C}_{N}}(\mathbf{r}), \\
f_{\mathrm{C}_{N} \mathrm{H}_{2 N+2}}^{-}(\mathbf{r})=\rho_{\mathrm{C}_{N} \mathrm{H}_{2 N+2}}(\mathbf{r})-\rho_{\left(\mathrm{C}_{N} \mathrm{H}_{2 N+2}\right)^{+}}(\mathbf{r})=\Delta \rho_{\mathrm{C}_{N} \mathrm{H}_{2 N+2}}(\mathbf{r}) .
\end{gathered}
$$

Regions where this function is large are especially reactive towards electrophiles; that is, electrophiles withdraw electronic charge preferentially from those regions. The electron density difference $\Delta \rho(\mathbf{r})$ reveals in Fig. 8 that the Fukui function $f_{\mathrm{C}_{N} \mathrm{H}_{2 N+2}}^{-}(\mathbf{r})$ shows substantial delocalization through the alkane chains. This delocalization is an effect common to all alkane sizes, and the spatial distribution of $f_{\mathrm{C}_{N} \mathrm{H}_{2 N+2}}^{-}(\mathbf{r})$ is similar in all of them. In contrast, the Fukui function of carbon clusters, $f_{\mathrm{C}_{N}}^{-}(\mathbf{r})$, presents differences when one compares different cluster sizes, as illustrated in the specific cases of $\mathrm{C}_{5}, \mathrm{C}_{7}$, and $\mathrm{C}_{9}$ in Fig. 8. 
a)

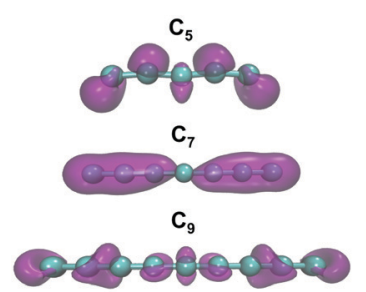

b)
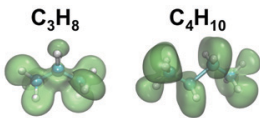

$\mathrm{C}_{8} \mathrm{H}_{18}$

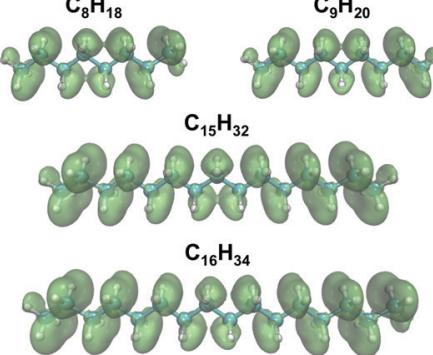

Fig. 8 Surfaces with a constant value (equal to $0.02 \mathrm{e} \AA^{-3}$ ) of the Fukui functions $f_{\mathrm{C}_{N}}^{-}(\mathbf{r})$ and $f_{\mathrm{C}_{N} \mathrm{H}_{2 N+2}}^{-}(\mathbf{r})$ for several carbon clusters and alkanes. The spatial distribution of the Fukui functions is measured by the difference between the electron densities of the corresponding neutral and cationic species. The regions where $f^{-}(\mathbf{r})$ is localized are regions prone to release electronic charge when the molecule reacts with electrophiles.

\subsection{Trends in the properties of each $\mathrm{C}_{N} \mathrm{H}_{n}$ family as $n$} increases. The road to saturation

Although our main purpose, developed in the previous sections, consists in studying how the addition of hydrogen affects the size effects in $\mathrm{C}_{N}$ clusters, that is, the variation of the properties as the number of $\mathrm{C}$ atoms in the cluster increases, a complementary but different question that we have also investigated is how the properties of $\mathrm{C}_{N}$ clusters of fixed $N$ change as hydrogen is added until full saturation is reached in the alkanes. For this purpose, the two representative families $\mathrm{C}_{5} \mathrm{H}_{n}(n=1-12)$ and $\mathrm{C}_{7} \mathrm{H}_{n}$ ( $n=1-16)$ have been selected. For each cluster $\mathrm{C}_{5} \mathrm{H}_{n}$ or $\mathrm{C}_{7} \mathrm{H}_{n}$ an extensive set of isomers have been considered and the lowest energy structure has been determined in each case. Fig. 9 shows the energies required to remove a single $\mathrm{H}$ atom from the $\mathrm{C}_{N} \mathrm{H}_{n}$ clusters $(n=5,7)$, which we call hydrogen evaporation energy for consistency with the notation used in other sections:

$$
E_{\mathrm{v}}\left(\mathrm{C}_{N} \mathrm{H}_{n}\right)=E\left(\mathrm{C}_{N} \mathrm{H}_{n-1}\right)+E(\mathrm{H})-E\left(\mathrm{C}_{N} \mathrm{H}_{n}\right),
$$

where the energies on the right hand side of the equation are the energies of the corresponding molecules and the energy of an isolated $\mathrm{H}$ atom, respectively. In the $\mathrm{C}_{5} \mathrm{H}_{n}$ family, an evenodd effect appears in the evaporation energy for molecules with $n$ between 6 and 12, where molecules with even $n$ have larger evaporation energies than molecules with odd $n$. Those differences arise from the details of the chemical bonding in the different molecules. Those details can be appreciated with the help of Fig. 10, which shows the calculated lowest energy structures of $\mathrm{C}_{5} \mathrm{H}_{n}$ with $n=1-12$.

$\mathrm{C}_{5} \mathrm{H}_{12}$ is a non-cyclic saturated hydrocarbon, in which all the $\mathrm{C}-\mathrm{C}$ bonds are single bonds. The molecule is very stable and consequently the energy to remove a single $\mathrm{H}$ atom is large, $E_{\mathrm{v}}\left(\mathrm{C}_{5} \mathrm{H}_{12}\right)=4.54 \mathrm{eV}$. After removing one $\mathrm{H}$ atom, the molecule obtained, $\mathrm{C}_{5} \mathrm{H}_{11}$, contains one unsaturated $\mathrm{C}$ orbital, which indicates that $\mathrm{C}_{5} \mathrm{H}_{11}$ is highly reactive. In fact, this molecule is only observed in extreme conditions. ${ }^{34}$ The low stability of this molecule is reflected in a low value of the energy to remove an

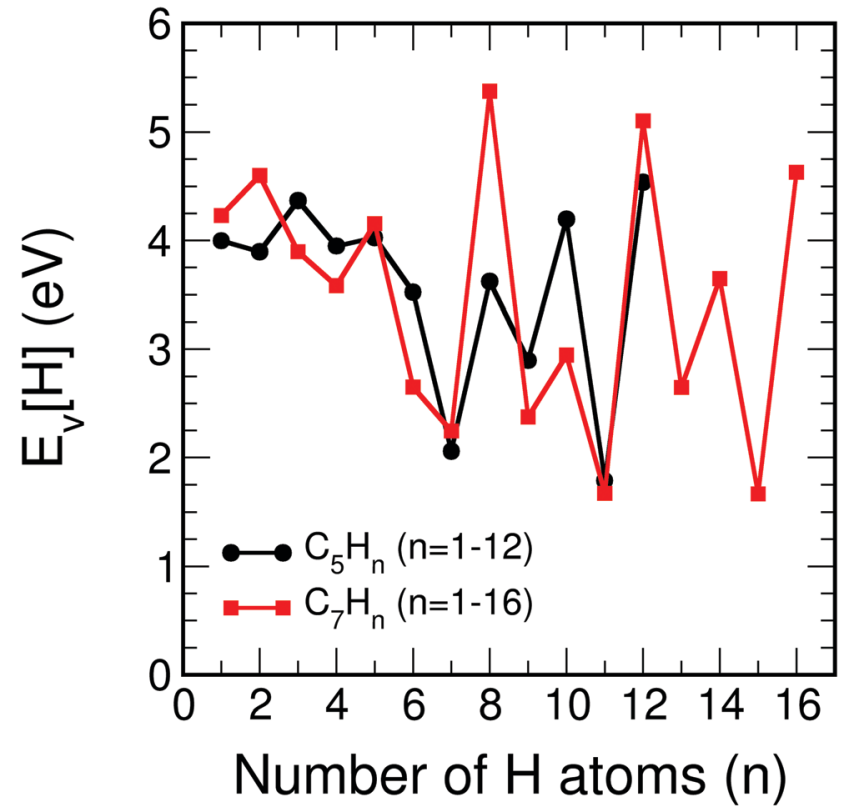

Fig. 9 Energy to remove an $\mathrm{H}$ atom, $E_{\mathrm{v}}$ (in $\mathrm{eV}$ ), as a function of the number of hydrogen atoms, $n$, from the $\mathrm{C}_{5} \mathrm{H}_{n}(n=1-12)$ and $\mathrm{C}_{7} \mathrm{H}_{n}(n=1-16)$ molecules.
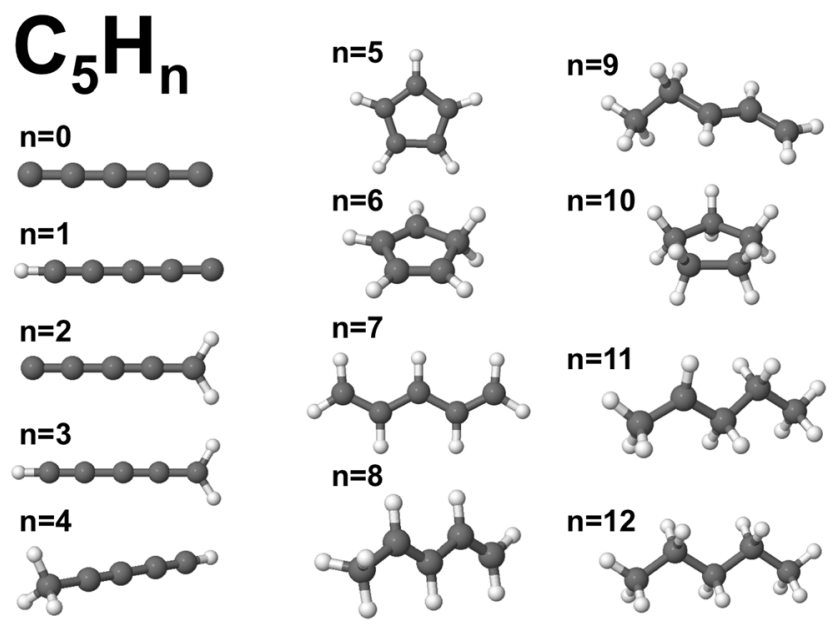

$n=10$

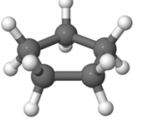

$\mathrm{n}=7$
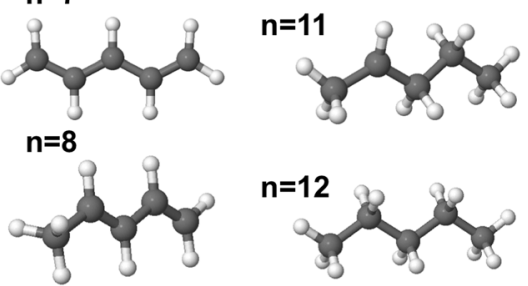

Fig. 10 Calculated ground state structures of $\mathrm{C}_{5} \mathrm{H}_{n}(n=0-12)$ molecules.

$\mathrm{H}$ atom, $E_{\mathrm{v}}=1.79 \mathrm{eV} . \mathrm{C}_{5} \mathrm{H}_{10}$ is a symmetric cyclic molecule with saturated bonds, which makes the molecule quite stable, with large $E_{\mathrm{v}}=4.20 \mathrm{eV}$. Loss of an $\mathrm{H}$ atom leads to $\mathrm{C}_{5} \mathrm{H}_{9}$, with an unsaturated $\mathrm{C}$ orbital. This molecule is, then, reactive and removing an $\mathrm{H}$ atom only costs an energy $E_{\mathrm{v}}=2.90 \mathrm{eV} \cdot \mathrm{C}_{5} \mathrm{H}_{8}$, with all the $\mathrm{C}$ bonds saturated, has a larger evaporation energy, $E_{\mathrm{v}}=3.62 \mathrm{eV} \cdot \mathrm{C}_{5} \mathrm{H}_{7}$ has a $\mathrm{C}$ atom with a non-saturated bond, and its higher reactivity leads to a low $E_{\mathrm{v}}=2.06 \mathrm{eV} . \mathrm{C}_{5} \mathrm{H}_{6}$ has all C bonds saturated, and $E_{\mathrm{v}}$ increases up to a value of $3.52 \mathrm{eV}$. In summary, the even-odd oscillations in the magnitude of $E_{\mathrm{v}}$ for $\mathrm{C}_{5} \mathrm{H}_{n}$ in the range $n=6-12$ are correlated with the presence or absence of unsaturated carbon orbitals. Local maxima of $E_{\mathrm{v}}$ occur for molecules with all bonds saturated $(n=6,8,10,12)$, while the local minima correspond to molecules with an 

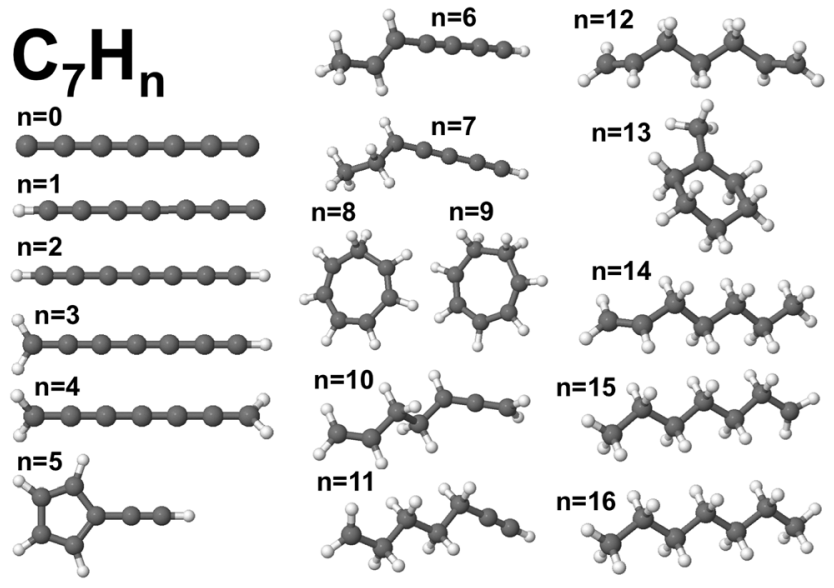

Fig. 11 Calculated ground state structures of $\mathrm{C}_{7} \mathrm{H}_{n}(n=1-16)$ molecules.

unsaturated C orbital $(n=7,9,11)$. The behavior is different in the region between $n=1$ and $n=5$, where all the evaporation energies are larger than $3.9 \mathrm{eV}$, in spite of the fact that all these molecules, except $\mathrm{C}_{5} \mathrm{H}_{4}$, have unsaturated $\mathrm{C}$ orbitals and are reactive. A similar trend in the hydrogen evaporation energies occurs in the $\mathrm{C}_{7} \mathrm{H}_{n}$ family, whose ground state structures are shown in Fig. 11. The even-odd oscillation shown in Fig. 9, that is, the correlation between local maxima of $E_{\mathrm{v}}$ and full bond saturation applies between $n=6$ and $n=16$, and $E_{\mathrm{v}}$ is again relatively large between $n=1$ and $n=5$. The trend of $E_{\mathrm{v}}$ as the number of $\mathrm{H}$ atoms in the molecule increases, starting with $\mathrm{C}_{N} \mathrm{H}$ and ending with the fully saturated molecule $\mathrm{C}_{N} \mathrm{H}_{2 N+2}$, is non smooth. However, as shown in the previous sections, after the $\mathrm{C}$ clusters are fully saturated with hydrogen, that is, when saturated alkanes are formed, the properties vary very smoothly with the alkane size, that is, with the number of $\mathrm{CH}_{2}$ groups added.

The ionization potentials and the electron affinities of $\mathrm{C}_{5} \mathrm{H}_{n}$ $(n=1-12)$ and $\mathrm{C}_{7} \mathrm{H}_{n}(n=1-16)$ have been plotted in Fig. 12. Similar to the behavior of $E_{\mathrm{v}}$ discussed above, IP shows large amplitude even-odd oscillation in each family between $n=6$ and $n=2 N$ : molecules with even values of $n$ have larger ionization potentials than molecules with odd values of $n$. As mentioned above, the molecules with even values of $n$ have all carbon orbitals saturated, while molecules with odd values of $n$ have one unsaturated bond. The even-odd oscillation is also quite convincing for $n$ smaller than 6 , with some misfit in the transition between the two regions. A complementary way of interpreting the even-odd oscillations in IP is in terms of spin pairing, since molecules with even $n$ have even number of electrons $(4 N+n$, excluding the core electrons of the carbon atoms). Even-odd oscillations of IP are also well known in alkali metal clusters. ${ }^{3,35}$ In spite of the sizable oscillations of IP shown in Fig. 12, two observations can be made. The values of IP for the molecules at the end of each family, $\operatorname{IP}\left(\mathrm{C}_{5} \mathrm{H}_{12}\right)=$ $9.93 \mathrm{eV}$ and $\mathrm{IP}\left(\mathrm{C}_{7} \mathrm{H}_{16}\right)=9.52 \mathrm{eV}$, are larger than the IPs of other members of the family, with the only exception of $\mathrm{C}_{5} \mathrm{H}_{10}$ in the $\mathrm{C}_{5} \mathrm{H}_{n}$ family. Second, the values of $\operatorname{IP}\left(\mathrm{C}_{5} \mathrm{H}_{12}\right)$ and $\operatorname{IP}\left(\mathrm{C}_{7} \mathrm{H}_{16}\right)$ are very close, which explains the trend observed in Fig. 7, that is,

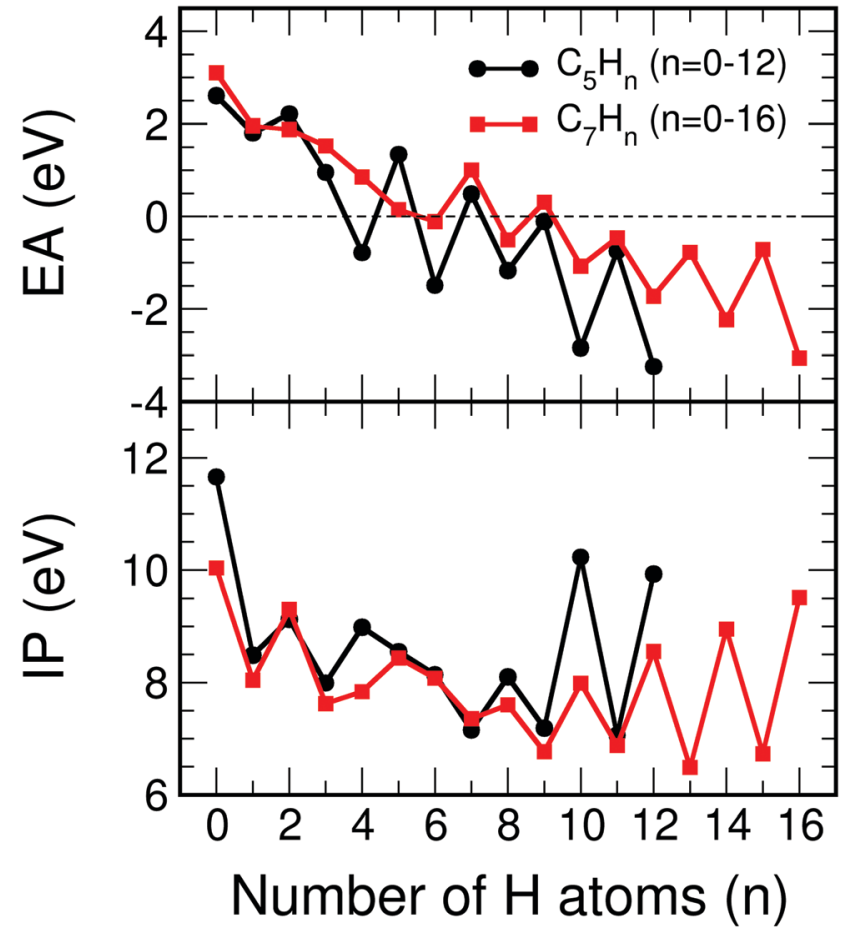

Fig. 12 Electron affinity, EA (in eV), and ionization potential, IP (in eV), as a function of the number of hydrogen atoms, $n$, of $\mathrm{C}_{5} \mathrm{H}_{n}(n=0-12)$ and $\mathrm{C}_{7} \mathrm{H}_{n}$ $(n=0-16)$ molecules (see structures in Fig. 10 and 11).

the quenching of size effects discussed in the first part of this work.

The electron affinities also show even-odd oscillations, between $n=3$ and $n=12$ in the $\mathrm{C}_{5} \mathrm{H}_{n}$ series, and between $n=5$ and $n=16$ in the $\mathrm{C}_{7} \mathrm{H}_{n}$ series. The oscillations confirm the spin paring, because the maxima of EA occur for clusters with odd number of electrons, where capture of one electron leads to an even number. However, the most important feature is that the electron affinity changes sign in the middle of each family. That is, EA becomes negative, which indicates that those molecular anions are not stable against electron detachment. In spite of this fact it is also worth to notice that the electron affinities of $\mathrm{C}_{5} \mathrm{H}_{12}$ and $\mathrm{C}_{7} \mathrm{H}_{16}$ are the most negative ones in their respective families, and that their values are nearly the same, supporting again the quenching of size effects in the saturated alkanes.

\section{Summary and conclusions}

The most fascinating characteristic of small atomic clusters is that the values of most of their properties do not scale smoothly with the cluster size $N$. Instead, the values of those properties can vary substantially in this regime in which every atom counts. This has been illustrated here by analyzing several properties of neutral $\mathrm{C}_{N}$ and cationic $\mathrm{C}_{N}^{+}$carbon clusters. The cluster structure changes from linear to cyclic at about $\mathrm{C}_{10}$. The $\mathrm{C}-\mathrm{C}$ bond lengths display an oscillating and irregular variation with the cluster size $N$. The cluster stability (related to the 
abundance measured in experiments of cluster formation in the gas phase) shows oscillations between highly stable and less stable clusters with increasing $N$. Also the variation of the ionization energy with $N$ deviates from a smooth behavior. Finally, the spatial distribution of the Fukui functions $f^{-}(\mathbf{r})$ (which reveal the regions more prone to realease electronic charge when the clusters react with electrophiles) shows differences between carbon clusters of different sizes. However, clusters can lose the size dependence of their properties by interaction with other chemical species. We have analyzed the case of carbon clusters fully saturated with hydrogen to form linear alkanes, $\mathrm{C}_{N} \mathrm{H}_{2 N+2}$, with sizes up to $N=25$. In all cases, the lowest energy structures are linear chains, the variations of $\mathrm{C}-\mathrm{C}$ bond lengths and $\widehat{\mathrm{CCC}}$ angles are minor and smooth, the stability functions show practically no structure as a function of alkane size, the ionization energies show no relevant features and decrease smoothly with alkane size, and the spatial distribution of the Fukui functions is analogous in all the linear alkanes. In summary, the interaction of carbon clusters with hydrogen to form alkanes quenches all the size-dependent features originally owned by the carbon clusters. The road to arrive at the quenching of the size effects is intricate. In each $\mathrm{C}_{N} \mathrm{H}_{n}$ family of fixed $N$, the values of the properties of the molecules like the ionization potential, the electron affinity, and others show sizable oscillations as the number $n$ of hydrogen atoms grows from the pure carbon cluster, where $n=0$, to the alkane, where $n=2 N+2$.

\section{Conflicts of interest}

The authors declare no competing financial interests.

\section{Acknowledgements}

We acknowledge funding from the Spanish MINECO (Grants MAT2017-85089-C2-1-R and RYC-2015-17730), Comunidad de Madrid (FotoArt-CM Project P2018/NMT-4367) through the Program of R\&D activities between research groups in Technologies 2018, co-financed by European Structural Funds, Junta de Castilla y León (Grant VA021G18), University of Valladolid (GIR Grupo de Física de Nanoestructuras), and the EU via the ERC-Synergy Program (Grant ERC-2013-SYG-610256 Nanocosmos) and EU Horizon 2020 Research and Innovation program (Grant 785219 - Graphene Flagship Core2-). We also thank the computing resources from CTI-CSIC.

\section{Notes and references}

1 E. Oger, N. Crawford, R. Kelting, P. Weis, M. Kappes and R. Ahlrichs, Angew. Chem., Int. Ed., 2007, 46, 8503-8506.

2 D. Emin, Phys. Today, 1987, 40, 56-62.

3 W. A. Saunders, K. Clemenger, W. A. de Heer and W. D. Knight, Phys. Rev. B: Condens. Matter Mater. Phys., 1985, 32, 1366-1368.
4 W. D. Knight, K. Clemenger, W. A. de Heer, W. A. Saunders, M. Y. Chou and M. L. Cohen, Phys. Rev. Lett., 1984, 52, 2141-2143.

5 C. Baladrón and J. A. Alonso, Phys. B, 1988, 154, 73-81.

6 Y. Dai, T. J. Gorey, S. L. Anderson, S. Lee, S. Lee, S. Seifert and R. E. Winans, J. Phys. Chem. C, 2017, 121, 361-374.

7 B. von Issendorff and O. Cheshnovsky, Annu. Rev. Phys. Chem., 2005, 56, 549-580.

8 J. Pérez-Juste, M. A. Correa-Duarte and L. M. Liz-Marzán, Appl. Surf. Sci., 2004, 226, 137-143.

9 S. E. Apsel, J. W. Emmert, J. Deng and L. A. Bloomfield, Phys. Rev. Lett., 1996, 76, 1441-1444.

10 J. Bansmann, V. Senz, R. P. Methling, R. Röhlsberger and K. H. Meiwes-Broer, Mater. Sci. Eng., C, 2002, 19, 305-310.

11 T. Hayashi, K. Tanaka and M. Haruta, J. Catal., 1998, 178, 566-575.

12 Nanocatalysis, ed. U. Heiz and U. Landman, Springer, 2007. 13 L. Li, A. H. Larsen, N. A. Romero, V. A. Morozov, C. Glinsvad, F. Abild-Pedersen, J. Greeley, K. W. Jacobsen and J. K. Nørskov, J. Phys. Chem. Lett., 2013, 4, 222-226.

14 F. Calle-Vallejo, J. I. Martnez, J. M. Garca-Lastra, P. Sautet and D. Loffreda, Angew. Chem., Int. Ed., 2014, 53, 8316-8319.

15 T. P. Martin, Phys. Rep., 1996, 273, 199-241.

16 J. A. Alonso, Structure and Properties of Atomic Nanoclusters, Imperial College Press, London, 2nd edn, 2012.

17 A. Artaud, L. Magaud, K. Ratter, B. Gilles, V. Guisset, P. David, J. I. Martnez, J. A. Martn-Gago, C. Chapelier and J. Coraux, Nano Lett., 2018, 18, 4812-4820.

18 M. J. Frisch, G. W. Trucks, H. B. Schlegel, G. E. Scuseria, M. A. Robb, J. R. Cheeseman, G. Scalmani, V. Barone, B. Mennucci, G. A. Petersson, H. Nakatsuji, M. Caricato, X. Li, H. P. Hratchian, A. F. Izmaylov, J. Bloino, G. Zheng, J. L. Sonnenberg, M. Hada, M. Ehara, K. Toyota, R. Fukuda, J. Hasegawa, M. Ishida, T. Nakajima, Y. Honda, O. Kitao, H. Nakai, T. Vreven, J. A. Montgomery, Jr., J. E. Peralta, F. Ogliaro, M. Bearpark, J. J. Heyd, E. Brothers, K. N. Kudin, V. N. Staroverov, R. Kobayashi, J. Normand, K. Raghavachari, A. Rendell, J. C. Burant, S. S. Iyengar, J. Tomasi, M. Cossi, N. Rega, J. M. Millam, M. Klene, J. E. Knox, J. B. Cross, V. Bakken, C. Adamo, J. Jaramillo, R. Gomperts, R. E. Stratmann, O. Yazyev, A. J. Austin, R. Cammi, C. Pomelli, J. W. Ochterski, R. L. Martin, K. Morokuma, V. G. Zakrzewski, G. A. Voth, P. Salvador, J. J. Dannenberg, S. Dapprich, A. D. Daniels, Ã. Farkas, J. B. Foresman, J. V. Ortiz, J. Cioslowski and D. J. Fox, Gaussian-09 Revision E.01, Gaussian Inc., Wallingford CT, 2009.

19 A. D. Becke, J. Chem. Phys., 1993, 98, 5648-5652.

20 P. J. Stephens, F. J. Devlin, C. F. Chabalowski and M. J. Frisch, J. Phys. Chem., 1994, 98, 11623-11627.

21 T. H. Dunning, J. Chem. Phys., 1989, 90, 1007-1023.

22 J. I. Martnez and J. A. Alonso, Phys. Chem. Chem. Phys., 2018, 20, 27368-27374.

23 K. Fukui, Science, 1982, 218, 747-754.

24 J. I. Martnez, M. J. López and J. A. Alonso, J. Chem. Phys., 2005, 123, 074303. 
25 P. W. Ayers and R. G. Parr, J. Am. Chem. Soc., 2000, 122, 2010-2018.

26 P. W. Ayers, R. C. Morrison and R. K. Roy, J. Chem. Phys., 2002, 116, 8731-8744.

27 L. Belau, S. E. Wheeler, B. W. Ticknor, M. Ahmed, S. R. Leone, W. D. Allen, H. F. Schaefer and M. A. Duncan, J. Am. Chem. Soc., 2007, 129, 10229-10243.

28 S. B. H. Bach and J. R. Eyler, J. Chem. Phys., 1990, 92, 358-363.

29 R. Ramanathan, J. A. Zimmerman and J. R. Eyler, J. Chem. Phys., 1993, 98, 7838-7845.
30 J. Benedikt, S. Agarwal, D. Eijkman, W. Vandamme, M. Creatore and M. C. M. van de Sanden, J. Vac. Sci. Technol., A, 2005, 23, 1400-1412.

31 CRC Handbook of Chemistry and Physics, ed. D. R. Lide, CRC Press, Boca Raton, FL., 81st edn, 2000.

32 C. Cao and H. Yuan, J. Chem. Inf. Comput. Sci., 2002, 42, 667-672.

33 N. G. Gotts, G. von Helden and M. T. Bowers, Int. J. Mass Spectrom. Ion Processes, 1995, 149-150, 217-229.

34 J. Pacansky and A. Gutierrez, J. Phys. Chem., 1983, 87, 3074-3079. 35 W. A. de Heer, Rev. Mod. Phys., 1993, 65, 611-676. 